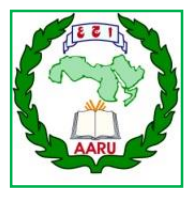

Arab Univ. J. Agric. Sci., Ain Shams Univ., Cairo, Egypt

29(1), 365 - 373, 2021

Website: http://ajs.journals.ekb.eg

DOI: 10.21608/ajs.2021.49621.1294

\title{
Obesity Assessments of Housewives in Great Cairo, Egypt
}

\section{Asmaa A Gabr ${ }^{2 *}$, Ramadan M Mahmoud², Mohamed F Khallaf ${ }^{2}$, Doaa H El Sabakhawi ${ }^{1}$}

1- National Institute of Nutrition, Cairo, Egypt

2- Food Sci. Dept, Fac of Agric, Ain Shams Univ, P.O. Box 68, Hadayek Shoubra, 11241, Cairo, Egypt

* Corresponding author: as.gabr1985@gmail.com

Received 23 December, 2020

Accepted 15 March, 2021

\begin{abstract}
A cross sectional study was conducted to evaluate obesity of housewives. The Sample that comprised of 200 females was selected from different regions of great Cairo, Egypt. Age was affected the BMI of various investigated obesity classes with a high correlation. A similar finding was also detected in case of education status where such parameter was also greatly affected the BMI status. The occupation, marital status and family size did not show any significant effect in BMI. The Income parameter didn't show any significant with the BMI. So, it could be concluded that only age and education status are greatly affected the housewives obesity in the investigated sample. Life style, i.e the sports practice. The type of sport, the time spent for sport practice, the place sports practice, household works making by herself, the number of times household works practice/week, the time of sleep in day and the time of last meal before sleeping were statistically significant with the BMI. Anthropometric measurements (waist circumference, waist-to-height ratio and central obesity) are greatly affecting the BMI meaning that there is a significant difference among various investigated obesity classes owing to such anthropometric factors. Regarding to the height parameter, no significant difference was found between overweight as well as obesity class I each other, and similar finding was also found
\end{abstract}

between obesity class 11 as well as obesity class III. The second variable (i.e., IBW \%) had no significant effect among the three obesity classes (I, II and III), while the overweight group was significantly differed rather than that of others. Considering the Central obesity variable, statistical analyses proved that no significance was detected with overweight or obesity class II group. Similar trend was also, noticed in case of (hip circumference) in relative to obesity class I and obesity class II.

Keywords: Obesity; Overweight; Anthropometric; Housewives; BMI; Demographic

\section{Introduction}

Obesity is one of the serious social and psychological problems, that virtually affects all age and socio-economic groups developing countries. The risk of obesity in developing countries is strongly influenced by diet and lifestyle, which dramatically changed as a result of the economic and nutritive conditions. Obesity is a risk factor in natural history of non-communicable diseases such as hypertension. Dietary life is the major element affecting health maintenance (WHO 2015). It is influenced by several environmental factors like age, job, education, economic level and family status (Chung et al 2011). 
The minimal researches that carried out so far on the difficulties in adolescents as well as children has shown only weak linear relationships between overweight and later diseases until made certain recommendations as international standard methods using for the measurement of body fat and obesity. So, cut-off points are easier to set for predicting co-morbidities like cardiovascular as well as hypertension diseases. Many studies were conducted through out1993-2007 in Egypt and revealed that obesity is a problem of public health that affecting various social as well as economic classes and different age groups. (Ezz El-Deen 2007).

Obesity means the excessive accumulation of fat which may impair health. The WHO indicates that body mass index (BMI) $\geq 30$ $\mathrm{kg} / \mathrm{m}^{2}$ considers as obese. Whereas, BMI between 25 and $29.9 \mathrm{~m}^{2}$ for adult is considered as overweight. According to the study confirmed that this syndrome is characterized by normal body weight and BMI, but high fat mass (>30\%) represents a risk factor for diabetes as well as cardiovascular disease. Obese women have a higher risk of type 2 diabetes mellitus in later life, the overweight was conducted to serious health risks for adults (Barma and Sil 2013). Nutrition is one of the most important factors influencing the quality of human life. Nutritional status is an important health indicator to assess a country's health status. The problem of world-wide obesity can be viewed as a consequence of the substantial social, cultural and economical problems that observed in developing and industrialized countries. It was influenced by four multiple factors, such as heredity, environment, personal behavior and access to other health services. Each group of housewives women had different life style and workloads for which they might have fitness level and different health status (Seerat and Shafia 2016).

This study was carried out to evaluate the nutritional status and obesity of housewives from different regions of great Cairo, Egypt to find a relationship between obesity and socioeconomic and demographic parameters age ,education status, occupation, marital status, family size, income, sport, homemaking, multimedia and sleeping as well as anthropometric measurements (Weight, height, waist-to-hip circumference ratio, IBW and WHtR) reaching to the best recommendations to reduce their obesity and to raise the nutritional and health status.

\section{Material and Methods}

\subsection{Sampling}

An cross sectional study was conducted to evaluate the nutritional status and obesity of housewives aged 20 to 60 years, Sample that comprised of 200 females were randomly selected from different regions of great Cairo, Egypt. Sampling was conducted through the medical convoy named (Hundred Million Health) in Cairo governorate during Oct. to Dec2018. These samples were stratified according to their body mass index (BMI) (Table 1).

Table 1. Classification of samples according to BMI

\begin{tabular}{|c|c|c|}
\hline BMI & No. & $\%$ \\
\hline $\begin{array}{l}\text { Overweight } 25-30 \\
\text { Healthy slightly increased }\end{array}$ & 17 & 8.5 \\
\hline $\begin{array}{l}\text { Obesity class I } 30-35 \\
\text { Healthy risk slightly increased }\end{array}$ & 65 & 32.5 \\
\hline $\begin{array}{l}\text { Obesity class II } 35-40 \\
\text { Healthy risk much increased }\end{array}$ & 57 & 28.5 \\
\hline $\begin{array}{l}\text { Obesity class III } \geq 40 \\
\text { Healthy risk very much increased }\end{array}$ & 61 & 30.5 \\
\hline Total & 200 & 100 \\
\hline
\end{tabular}

\subsection{Socio-economic and demographic pa- rameters}

For this study, questionnaire was designed as a way for collecting data, then data were recorded through personal interview. The questionnaire data revolve around four main axes: Demographic information that includes several questions like age, education status, marital status, occupation and family size and income. Lifestyle including sports, homemaking, multimedia and sleeping. 


\subsection{Anthropometric measurements}

Weight was taken using a digital weighing machine to the nearest $0.1 \mathrm{Kg}$. Height was recorded using an inch tape to the nearest of $0.1 \mathrm{~cm}$. Waist and hip circumference was also taken using inch tape to the nearest of $0.5 \mathrm{~cm}$. All measurements were taken with the respondents, barefoot. Nutritional status was assessed using BMI (body mass index), central obesity, IBW\% (ideal body weight), WHtR (Waist-to-Height Ratio) and body fat\%., Central obesity was checked in the respondents using waist-to-hip ratio, IBW= (real weight/perfect weight) $\mathrm{x} 100$, Waist -to-height ratio (WHtR) all measurements were performed according to the techniques described by Whitney et al (2010), Lee et al (2008).

\subsection{Statistical analysis}

The results obtained were analyzed using SPSS (the nineteenth version) and find mean, standard error and analyzing ANOVA one away, DUNCAN test, Chi-square and statistical significance level was $\leq 0.05$ according to (Steel et al 1997).

\section{Results and Discussion}

\subsection{Socio-economic and demographic pa- rameters}

The socioeconomic data that collected from investigation samples were given in Table 2 . Such data are age, education status, occupation, marital status, family size and income. Regarding to the age as an affecting factor on BMI which expresses about true obesity, it could be noticed that it was significantly affected the BMI of various investigated obesity classes with a high correlation ( $\mathrm{p}$-value= 0.005). A Similar finding that shown earlier was also detected in case of education status. It could be seen that $\mathrm{p}$-value was 0.031 , i.e. such parameter was also greatly affected the BMI status (Girdhar et al 2016). Another investigated parameters such as occupation, marital status and family size were also given in the same table such three parameters did not show any significant effect, i.e. p-value was around 0.06 . It had $0.771,0.545$ and 0.608 respectively. Income parameter didn't show any statistical significance with the BMI (p-value= 0.218 ). So, it could be concluded that only age and education status are greatly affected house wives obesity in the investigated sample (Farhood and Abbas 2015).

Life style of housewives in great Cairo was also studied to indicate the effect of life style classification on BMI Table 3. From this table it could be reported that the sports practice, The type of sport, the time spent of sport practice, the place sports practice, household works making by herself, the number of times household works practice/week, the time of sleep in day and the time of last meal before sleeping were statistically significant with the BMI of the housewives ( $\mathrm{p}$-value $=0.09,0.05,0.05$, $0.01,0.02,0.03,0.03$ and 0.03 ), respectively.

The time spent in household works /once as well as the time spent of watching TV or computer had a p-value equal 0.421 and .0490 , respectively. It means that such two items did not show any significance (Sen and Verma 2016)

\subsection{Anthropometric measurements}

Anthropometric measurements as affecting factors on BMI were given in Table 4. It could be seen that such factors (waist circumference, waist-to-height ratio and central obesity) are greatly affecting the BMI (p-value 0.00, 0.00 and 0.01 respectively) meaning that there is a significant difference between various investigated obesity classes owing to such anthropometric factor (Patel et al 2017).

Finally, Table 5 statistically indicates the role of variables in obesity classes of Egyptian housewives live in great Cairo. From this Table there is a significant difference among different obesity classes as affecting by most of these variables. Regarding to the height parameters, no significant difference was found between overweight as well as obesity class I each other, and a similar finding was also found between obesity class II as well as obesity class III. The second variable (i.e IBW\%) had no significant effect among three obesity 
Table 2. Socioeconomic measurements of housewives under studying as affecting factor on BMI

\begin{tabular}{|c|c|c|c|c|c|c|c|c|c|c|c|}
\hline \multirow{3}{*}{\multicolumn{2}{|c|}{ Variable }} & \multirow{2}{*}{\multicolumn{2}{|c|}{$\begin{array}{c}\text { Overweight } \\
(\mathbf{n}=17)\end{array}$}} & \multirow{2}{*}{\multicolumn{2}{|c|}{$\begin{array}{c}\text { Obesity } \\
\text { Class } 1 \\
(n=65)\end{array}$}} & \multirow{2}{*}{\multicolumn{2}{|c|}{$\begin{array}{c}\text { Obesity } \\
\text { Class } 11 \\
(n=57) \\
\end{array}$}} & \multirow{2}{*}{\multicolumn{2}{|c|}{$\begin{array}{c}\text { Obesity } \\
\text { Class } 111 \\
(n=61) \\
\end{array}$}} & \multirow{2}{*}{\begin{tabular}{|l|} 
P-value \\
\end{tabular}} & \multirow{2}{*}{\begin{tabular}{|l|} 
Chi-square \\
\end{tabular}} \\
\hline & & & & & & & & & & & \\
\hline & & & & & & & & & & & \\
\hline \multirow{5}{*}{ Age } & 20-29 years & 5 & 29.4 & 6 & 9.2 & 5 & 8.8 & 5 & 8.2 & \multirow{5}{*}{$0.005^{\mathrm{a}}$} & \multirow{5}{*}{28.444} \\
\hline & 30-39 years & 6 & 35.3 & 30 & 46.2 & 17 & 29.8 & 14 & 23.0 & & \\
\hline & $40-49$ years & 4 & 23.5 & 17 & 26.2 & 23 & 40.0 & 17 & 27.9 & & \\
\hline & $50-60$ years & 1 & 5.9 & 10 & 15.4 & 5 & 8.8 & 19 & 31.1 & & \\
\hline & $>60$ years & 1 & 5.6 & 2 & 3.1 & 7 & 12.3 & 6 & 9.3 & & \\
\hline \multirow{5}{*}{$\begin{array}{c}\text { Education sta- } \\
\text { tus }\end{array}$} & Illiterate & 2 & 11.8 & 8 & 12.3 & 12 & 21.1 & 17 & 27.9 & \multirow{5}{*}{$0.031^{\mathrm{a}}$} & \multirow{5}{*}{19.254} \\
\hline & Elementary & 0 & 0 & 3 & 4.6 & 0 & 0 & 5 & 8.2 & & \\
\hline & Preparatory & 0 & 0 & 1 & 1.5 & 4 & 7.0 & 4 & 6.6 & & \\
\hline & Intermediate & 11 & 64.7 & 32 & 49.2 & 22 & 38.6 & 23 & 37.7 & & \\
\hline & $\begin{array}{c}\text { University } \\
\text { Degree } \\
\end{array}$ & 4 & 23.5 & 21 & 32.3 & 19 & 33.3 & 12 & 19.7 & & \\
\hline \multirow{2}{*}{ Occupation } & working & 2 & 11.8 & 9 & 13.8 & 5 & 8.8 & 9 & 14.8 & \multirow{2}{*}{0.771} & \multirow{2}{*}{1.124} \\
\hline & Not working & 15 & 88.2 & 56 & 86.2 & 52 & 91.2 & 52 & 85.7 & & \\
\hline \multirow{4}{*}{ Marital Status } & Married & 12 & 70.6 & 47 & 72.3 & 45 & 78.9 & 40 & 65.6 & \multirow{4}{*}{0.545} & \multirow{4}{*}{7.893} \\
\hline & Divorced & 2 & 11.8 & 8 & 12.3 & 6 & 10.5 & 9 & 14.8 & & \\
\hline & Widower & 1 & 5.9 & 8 & 12.3 & 6 & 10.5 & 9 & 14.8 & & \\
\hline & Single & 2 & 11.8 & 2 & 3.1 & 0 & 0 & 3 & 4.9 & & \\
\hline \multirow{3}{*}{ Family size } & $\leq 3$ & 1 & 6.3 & 7 & 10.8 & 3 & 5.3 & 8 & 13.6 & \multirow{3}{*}{0.608} & \multirow{3}{*}{4.511} \\
\hline & $4-5$ & 10 & 62.5 & 36 & 55.4 & 39 & 68.4 & 31 & 52.5 & & \\
\hline & $>5$ & 5 & 31.3 & 22 & 33.8 & 15 & 26.3 & 20 & 33.9 & & \\
\hline \multirow{3}{*}{ Income } & LOW & 0 & 0 & 3 & 4.6 & 6 & 10.5 & 2 & 3.3 & \multirow{3}{*}{0.218} & \multirow{3}{*}{4.437} \\
\hline & Middle & 17 & 100.0 & 62 & 95.4 & 51 & 89.5 & 59 & 96.7 & & \\
\hline & High & 0 & 0 & 0 & 0 & 0 & 0 & 0 & 0 & & \\
\hline
\end{tabular}

$\mathrm{p}$-value $\leq 0.05$ is significant. 
Table 3. Life style parameters as affecting factors on BMI

\begin{tabular}{|c|c|c|c|c|c|c|c|c|c|c|c|}
\hline \multirow{2}{*}{\multicolumn{2}{|c|}{ Variable }} & \multicolumn{2}{|c|}{$\begin{array}{c}\text { Overweight } \\
(\mathbf{n}=17)\end{array}$} & \multicolumn{2}{|c|}{$\begin{array}{c}\text { Obesity } \\
\text { Class } 1 \\
(n=65)\end{array}$} & \multicolumn{2}{|c|}{$\begin{array}{c}\text { Obesity } \\
\text { Class11 } \\
(n=57)\end{array}$} & \multicolumn{2}{|c|}{$\begin{array}{c}\text { Obesity } \\
\text { Class111 } \\
(n=61)\end{array}$} & \multirow[b]{2}{*}{ P-value } & \multirow[b]{2}{*}{\begin{tabular}{|c} 
Chi- \\
square
\end{tabular}} \\
\hline & & No & $\%$ & No & $\%$ & No & $\%$ & No & $\%$ & & \\
\hline \multirow{2}{*}{ Sports } & Yes & 6 & 35.3 & 16 & 24.6 & 13 & 22.8 & 7 & 11.5 & \multirow{2}{*}{$0.099^{\mathrm{a}}$} & \multirow{2}{*}{6.054} \\
\hline & No & 11 & 64.7 & 49 & 75.4 & 44 & 77.2 & 54 & 88.5 & & \\
\hline \multirow{7}{*}{$\begin{array}{c}\text { Type of } \\
\text { sport }\end{array}$} & Aerobics & 11 & 64.4 & 49 & 75.4 & 44 & 77.2 & 54 & 88.5 & \multirow{7}{*}{$0.056^{\mathrm{a}}$} & \multirow{7}{*}{31.494} \\
\hline & Belly dance & 0 & 0 & 2 & 3.1 & 0 & 0 & 0 & 0 & & \\
\hline & Fitness & 2 & 11.8 & 1 & 1.5 & 1 & 1.8 & 0 & 0 & & \\
\hline & Gym & 2 & 11.8 & 2 & 3.1 & 5 & 5.3 & 2 & 3.3 & & \\
\hline & Walk & 0 & 0 & 3 & 4.6 & 3 & 5.3 & 5 & 8.2 & & \\
\hline & Yoga & 1 & 5.9 & 1 & 1.5 & 1 & 1.8 & 0 & 0 & & \\
\hline & Zomba & 1 & 5.9 & 3 & 4.6 & 1 & 1.8 & 0 & 0 & & \\
\hline \multicolumn{12}{|c|}{ If yes } \\
\hline \multirow{4}{*}{$\begin{array}{c}\text { Time spent } \\
\text { of sport }\end{array}$} & $30 \mathrm{~min}$. / once & 0 & 0 & 1 & 6.3 & 0 & 0 & 0 & 0 & \multirow{4}{*}{$0.0544^{\mathrm{a}}$} & \multirow{4}{*}{7.905} \\
\hline & $60 \mathrm{~min}$. / once & 6 & 100.0 & 14 & 87.5 & 11 & 84.6 & 7 & 100.0 & & \\
\hline & $120 \mathrm{~min} . /$ once & 0 & 0 & 0 & 0 & 2 & 15.4 & 0 & 0 & & \\
\hline & $180 \mathrm{~min} . /$ once & 0 & 0 & 1 & 6.3 & 0 & 0 & 0 & 0 & & \\
\hline \multirow{5}{*}{$\begin{array}{c}\text { Place of } \\
\text { sport }\end{array}$} & At home & 0 & 0 & 0 & 0 & 0 & 0 & 0 & 0 & \multirow{5}{*}{$0.018^{\mathrm{a}}$} & \multirow{5}{*}{10.063} \\
\hline & In the street & 0 & 0 & 3 & 18.8 & 3 & 23.1 & 5 & 71.4 & & \\
\hline & $\begin{array}{c}\text { The public } \\
\text { square }\end{array}$ & 0 & 0 & 0 & 0 & 0 & 0 & 0 & 0 & & \\
\hline & Gym & 6 & 100.0 & 13 & 81.3 & 10 & 76.9 & 2 & 28.6 & & \\
\hline & Others & 0 & 0 & 0 & 0 & 0 & 0 & 0 & 0 & & \\
\hline
\end{tabular}

p-value $\leq 0.05$ is significant. 
Table 3. Cont.

\begin{tabular}{|c|c|c|c|c|c|c|c|c|c|c|c|}
\hline \multirow{4}{*}{$\begin{array}{c}\text { che } \\
\text { Household } \\
\text { works making } \\
\text { by herself }\end{array}$} & & \multicolumn{2}{|c|}{$\begin{array}{l}\text { Overweight } \\
\quad(n=17)\end{array}$} & \multicolumn{2}{|c|}{$\begin{array}{l}\text { Obesity } \\
\text { Class } 1 \\
(n=65)\end{array}$} & \multicolumn{2}{|c|}{$\begin{array}{c}\text { Obesity } \\
\text { Class11 } \\
(n=57)\end{array}$} & \multicolumn{2}{|c|}{$\begin{array}{c}\text { Obesity } \\
\text { Class111 } \\
(n=61)\end{array}$} & \multirow[b]{2}{*}{ P-value } & \multirow[b]{2}{*}{$\begin{array}{l}\text { Chi- } \\
\text { square }\end{array}$} \\
\hline & & No & $\%$ & No & $\%$ & No & $\%$ & No & $\%$ & & \\
\hline & Yes & 15 & 88.2 & 53 & 81.5 & 48 & 84.2 & 39 & 63.9 & \multirow[b]{2}{*}{$0.022^{\mathrm{a}}$} & \multirow[b]{2}{*}{9.641} \\
\hline & No & 2 & 11.8 & 12 & 18.5 & 9 & 15.8 & 22 & 36.1 & & \\
\hline \multicolumn{12}{|c|}{ If yes } \\
\hline \multirow{4}{*}{$\begin{array}{c}\text { The number of } \\
\text { times household } \\
\text { works/week }\end{array}$} & 2 time / week & 0 & 0 & 1 & 1.9 & 2 & 4.2 & 5 & 12.8 & \multirow{4}{*}{$0.030^{\mathrm{a}}$} & \multirow{4}{*}{18.512} \\
\hline & 3 time / week & 1 & 6.7 & 7 & 13.2 & 1 & 2.1 & 5 & 12.8 & & \\
\hline & 5 time / week & 4 & 26.7 & 5 & 9.4 & 4 & 8.3 & 1 & 2.6 & & \\
\hline & 7 time / week & 10 & 66.7 & 40 & 75.5 & 41 & 85.4 & 28 & 71.8 & & \\
\hline \multirow{3}{*}{$\begin{array}{c}\text { Time spent in } \\
\text { household works } \\
\text { / everyone }\end{array}$} & $60 \mathrm{~min} . /$ once & 8 & 53.3 & 20 & 37.7 & 24 & 50.0 & 22 & 56.4 & \multirow{3}{*}{0.421} & \multirow{3}{*}{9.175} \\
\hline & $\begin{array}{c}180 \text { min. / } \\
\text { once }\end{array}$ & 0 & 0 & 4 & 7.5 & 0 & 0 & 3 & 7.7 & & \\
\hline & $\begin{array}{c}240 \text { min. I } \\
\text { once }\end{array}$ & 1 & 6.7 & 2 & 3.8 & 2 & 4.2 & 2 & 5.1 & & \\
\hline \multirow{5}{*}{$\begin{array}{l}\text { The time spent } \\
\text { of watching TV, } \\
\text { playing on com- } \\
\text { puter... }\end{array}$} & 0 & 3 & 17.6 & 12 & 18.5 & 12 & 21.1 & 16 & 26.2 & \multirow{5}{*}{0.490} & \multirow{5}{*}{11.461} \\
\hline & 1 hour/day & 0 & 0 & 4 & 6.2 & 7 & 12.3 & 5 & 8.2 & & \\
\hline & 2 hour/day & 6 & 35.3 & 17 & 26.2 & 15 & 26.3 & 9 & 14.8 & & \\
\hline & 3 hour/day & 5 & 29.4 & 14 & 21.5 & 13 & 22.8 & 11 & 18.0 & & \\
\hline & 5 hour/day & 3 & 17.6 & 18 & 27.7 & 10 & 17.5 & 20 & 32.8 & & \\
\hline \multirow{4}{*}{$\begin{array}{l}\text { The time of } \\
\text { sleep in day }\end{array}$} & 0 & & & & & & & & & \multirow{4}{*}{$0.039^{\mathrm{a}}$} & \multirow{4}{*}{11.302} \\
\hline & 1 hour/day & 1 & 9.1 & 4 & 12.1 & 4 & 11.4 & 8 & 20.5 & & \\
\hline & 2 hour/day & 7 & 63.6 & 28 & 84.8 & 24 & 68.6 & 20 & 51.3 & & \\
\hline & 3 hour/day & 3 & 27.3 & 1 & 3.0 & 7 & 20.0 & 11 & 28.2 & & \\
\hline \multirow{3}{*}{$\begin{array}{l}\text { The time of } \\
\text { sleep in night }\end{array}$} & $\leq 3$ hour/day & 0 & 0 & 1 & 1.5 & 1 & 1.8 & 0 & 0 & \multirow{3}{*}{0.810} & \multirow{3}{*}{12.680} \\
\hline & 4 - 6 hour/day & 3 & 17.6 & 5 & 7.7 & 10 & 17.5 & 15 & 24.6 & & \\
\hline & $\geq 6$ hour/day & 14 & 82.3 & 59 & 90.8 & 46 & 80.8 & 46 & 75.4 & & \\
\hline \multirow{3}{*}{$\begin{array}{l}\text { The time of the } \\
\text { last meal before } \\
\text { sleeping }\end{array}$} & Before 9 pm & 3 & 17.6 & 14 & 21.5 & 17 & 29.8 & 12 & 19.7 & \multirow{3}{*}{$0.0351^{\mathrm{a}}$} & \multirow{3}{*}{6.683} \\
\hline & $\begin{array}{c}\text { From } 9-12 \\
\text { pm }\end{array}$ & 14 & 82.4 & 51 & 78.5 & 40 & 70.2 & 47 & 77.0 & & \\
\hline & After 12 Am & 0 & 0 & 0 & 0 & 0 & 0 & 2 & 3.3 & & \\
\hline
\end{tabular}

p-value $\leq 0.05$ is significant. 
Table 4. Anthropometric measurements as affecting factor on BMI

\begin{tabular}{|c|c|c|c|c|c|c|c|c|c|c|c|}
\hline \multirow{2}{*}{\multicolumn{2}{|c|}{ Variable }} & \multicolumn{2}{|c|}{$\begin{array}{l}\text { Over- } \\
\text { weight } \\
(n=17)\end{array}$} & \multicolumn{2}{|c|}{$\begin{array}{l}\text { Obesity } \\
\text { Class } 1 \\
(n=65)\end{array}$} & \multicolumn{2}{|c|}{$\begin{array}{c}\text { Obesity } \\
\text { Class11 } \\
(n=57)\end{array}$} & \multicolumn{2}{|c|}{$\begin{array}{c}\text { Obesity } \\
\text { Class111 } \\
(n=61)\end{array}$} & \multirow[b]{2}{*}{$\begin{array}{c}\mathbf{P}- \\
\text { value }\end{array}$} & \multirow[b]{2}{*}{$\begin{array}{l}\text { Chi- } \\
\text { square }\end{array}$} \\
\hline & & No & $\%$ & No & $\%$ & No & $\%$ & No & $\%$ & & \\
\hline \multirow{3}{*}{$\begin{array}{c}\text { Waist } \\
\text { Circumference }\end{array}$} & $\begin{array}{c}\text { Normal } \\
(64-80 \mathrm{~cm})\end{array}$ & 10 & 58.8 & 13 & 20.0 & 4 & 7.0 & 1 & 1.6 & \multirow{3}{*}{$0.000^{\mathrm{a}}$} & \multirow{3}{*}{58.475} \\
\hline & $\begin{array}{l}\text { Elevated risk } \\
(80-88 \mathrm{~cm})\end{array}$ & 3 & 17.6 & 15 & 23.1 & 9 & 15.8 & 1 & 1.6 & & \\
\hline & $\begin{array}{l}\text { High risk }(> \\
88 \mathrm{~cm})\end{array}$ & 4 & 23.5 & 37 & 56.9 & 44 & 77.2 & 59 & 96.7 & & \\
\hline \multirow{3}{*}{$\begin{array}{l}\text { Waist-to- } \\
\text { height ratio } \\
\text { WHtR }\end{array}$} & $\begin{array}{c}\text { No increased } \\
\text { risk }<.5\end{array}$ & 2 & 50 & 1 & 25 & 0 & 0 & 1 & 34.7 & \multirow{3}{*}{$0.000^{\mathrm{a}}$} & \multirow{3}{*}{45.521} \\
\hline & $\begin{array}{c}\text { Increased } \\
\text { risk } 0.5:<.6\end{array}$ & 8 & 34.8 & 12 & 52.2 & 3 & 13 & 0 & 0 & & \\
\hline & $\begin{array}{l}\text { Very high } \\
\text { risk } \geq .6\end{array}$ & 7 & 4 & 52 & 30.1 & 54 & 31.2 & 60 & 34.7 & & \\
\hline \multirow{2}{*}{$\begin{array}{l}\text { Central obe- } \\
\text { sity }\end{array}$} & Normal & 12 & 70.6 & 46 & 70.8 & 30 & 52.6 & 23 & 37.7 & \multirow{2}{*}{$0.001^{\mathrm{a}}$} & \multirow{2}{*}{15.714} \\
\hline & Obesity & 5 & 29.4 & 19 & 29.2 & 27 & 47.4 & 38 & 62.3 & & \\
\hline
\end{tabular}

1 -p-value $\leq 0.05$ is significant

Table 5. The role of variables in obesity classes of Egyptian housewives

\begin{tabular}{|l|c|c|c|c|}
\hline \multicolumn{1}{|c|}{ Variable } & $\begin{array}{c}\text { Over } \\
\text { weight } \\
(\mathbf{n = 1 7 )}\end{array}$ & $\begin{array}{c}\text { Obesity } \\
\text { Class } \\
\text { I(n= 65) }\end{array}$ & $\begin{array}{c}\text { Obesity } \\
\text { Class } \\
\text { II(n=57) }\end{array}$ & $\begin{array}{c}\text { Obesity } \\
\text { Class } \\
\text { III(n=61) }\end{array}$ \\
\hline Weight, kg & $72.4 \pm 2.18^{\mathrm{d}^{*}}$ & $81.3 \pm .86^{\mathrm{c}}$ & $90.1 \pm 1.05^{\mathrm{b}}$ & $108.1 \pm 1.9^{\mathrm{a}}$ \\
\hline Age & $37.3 \pm 2.5^{\mathrm{d}}$ & $40.6 \pm 1.1^{\mathrm{cd}}$ & $43.6 \pm 1.3^{\mathrm{bc}}$ & $46.2 \pm 0.7^{\mathrm{a}}$ \\
\hline Height, cm & $158.7 \pm 1.84^{\mathrm{a}}$ & $158.4 \pm 0.7^{\mathrm{a}}$ & $155.7 \pm 0.8^{\mathrm{ab}}$ & $153.08 \pm 0.7^{\mathrm{ab}}$ \\
\hline BMI, kg/m ${ }^{2}$ & $28.7 \pm 0.2^{\mathrm{d}}$ & $32.3 \pm 0.2^{\mathrm{c}}$ & $37.1 \pm 0.2^{\mathrm{b}}$ & $46.2 \pm 0.8^{\mathrm{a}}$ \\
\hline IBW, \% & $74.6 \pm 0.1^{\mathrm{b}}$ & $75.7 \pm 0.2^{\mathrm{a}}$ & $76.2 \pm 0.2^{\mathrm{a}}$ & $76.04 \pm 0.2^{\mathrm{a}}$ \\
\hline Waist circumference, cm & $95.6 \pm 3.8^{\mathrm{d}}$ & $103.3 \pm 1.4^{\mathrm{c}}$ & $110.04 \pm 1.5^{\mathrm{b}}$ & $127.5 \pm 1.6^{\mathrm{a}}$ \\
\hline Waist-to-Height Ratio $(\mathrm{WHtR}), \mathrm{cm}$ & $0.6 \pm 0.02^{\mathrm{d}}$ & $0.6 \pm 0.5^{\mathrm{c}}$ & $0.7 \pm 0.009^{\mathrm{b}}$ & $0.9 \pm 0.1^{\mathrm{a}}$ \\
\hline Central obesity,cm & $0.8 \pm 0.01^{\mathrm{b}}$ & $0.8 \pm .007^{\mathrm{ab}}$ & $0.80 \pm .04^{\mathrm{b}}$ & $0.80 \pm .01^{\mathrm{a}}$ \\
\hline Body fat \% & $26.9 \pm 0.6^{\mathrm{d}}$ & $32.01 \pm 0.2^{\mathrm{c}}$ & $38.3 \pm 0.3^{\mathrm{b}}$ & $49.8 \pm 1.04^{\mathrm{a}}$ \\
\hline Hip circumference, cm & $118.2 \pm 3.1^{\mathrm{b}}$ & $125.4 \pm 0.9^{\mathrm{ab}}$ & $128.3 \pm 1.3^{\mathrm{ab}}$ & $170.2 \pm 23.02^{\mathrm{a}}$ \\
\hline
\end{tabular}

* Data are mean \pm SE, means with the same letter in the same raw are not significantly different $\mathrm{p}<0.05$.

classes (I, II and III), while overweight group was significantly differed rather than that of others. Considering the central obesity variable, statistical analyses proved that no significantly between overweight or obesity class II groups. A Similar trend was also noticed in case of variable, that named hip circumference but such insignificance was existed between obesity class 1 and obesity class 11 .

\section{Conclusion}

This study based on random sample (200) of housewives of great Cairo Age and education status were significantly affected the BMI of various investigated obesity classes (I, II and III). Another investigated parameters such as occupation, marital status, family size and income were also given. such three parameters did not show any significant effect. So, it could 
be concluded that only age and education status are greatly affected house wives obesity. Sports, homemaking, multimedia and sleeping were statistically significant with the BMI of the housewives. Anthropometric parameters were greatly affected housewives obesity.

\section{References}

Barma, SR; Sil, P (2013) A comparative study of health and nutritional status among housewives and working women of north Bengal. International Journal of Behavioral Social and Movement Sciences, 2, 35-40.

Chung, K; Shin, K, Yoon, JK; Choi, KS (2011) Study on the obesity and nutrition status of housewives in Seoul and Kyunggi area. Nutrition Research and Practice, 5, 140-149.

Ezz El-Deen, MM (2007) Food consumption Pattern in relation to obesity in Egypt. M.Sc. Thesis Fac Agric, Ain Shams Univ, Cairo, Egypt.

Farhood, HF; Abbas, RA (2015) Association of dietary intake and eating habit with risk of pre diabetes in postmenopausal women. Iraqi Journal of Community Medicine 28, 173-178.

Girdhar, S; Sharma, S; Chaudhary, A; Bansal, P; Mahesh, S (2016) An Epidemiological study of overweight and obesity among women in an urban area of north India. Indian Journal of Community Medicine 41, 154-157.

Lee, CM; Huxley RR; Wildman, RP; Woodward, M (2008) Indices of abdominal obesity are better discriminators of cardiovascular risk factors than BMI: A meta-analsis. J. Clin. Epidemiol 61, 646-653.

Patel, AP; Patel, PP; Yagnik, B; Vora, K; Verma, A; Patel, AD; Chiplonkar, SA; Khadilkar, AV (2017) Nutritional status assessment of women from different occupation in urban and semi-urban regions of Gujarat .International Journal of Food and Nutritional Science 3, 381-384.

Seerat; Majied, S; Shafia, S (2016) Nutrition and food consumption of Non-working women in Kashmir (Rural \& Urban). International Journal of Information Research and Review 3, 3269-3272.

Sen, S; Verma, R (2016) Assessment of nutritional status of urban women of Maharashtra, India. Iosr Journal of Nursing and Health Science (IOSR-JNHS), 5, 1-7.

Steel, R; Torrie, J; Dickey, D (1997) Principles and procedures of statistics: A Biometrical Approach. $3^{\text {rd }}$ Ed, McGraw-Hill, New York, USA.

Whitney, E; Kelly, DL; Pinna, K; Rady, RS (2010) Nutrition for health and health care. Cen Gage Learning 4, 394-395.

WHO (2000) World Health Organization. http://www.toybox-study.eu/?q=de/node/31.

WHO (2015) World Health Organization. Obesity and Overweight. Last accessed on April 25, 2015. 


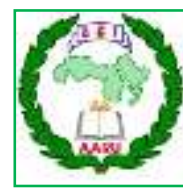

مجلة اتحاد الجامعات العربية للطوم الزراعية، جامعة عين شمس، القاهرة، مصر

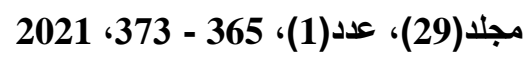

Website: http://ajs.journals.ekb.eg

DOI: 10.21608/ajs.2021.49621.1294

تقييم حالات السمنة لربات البيوت بالقاهرة الكبري-مصر

[24]

أسماء عبدالرحمن جبر2" - رمضان محمد محمود2 - محمد فرج خلاف2 - دعاء حامد السبخاوي1 1

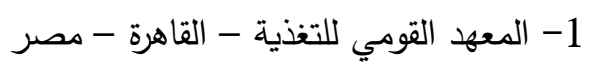

2- قسم علوم الأغذية - كلية الزراعة - جامعة عين شمس - ص.ب 68 - حدائق شبرا 11241 - القاهرة -

* Corresponding author: as.gabr1985@gmail.com

Received 23 December, 2020

Accepted 15 March, 2021

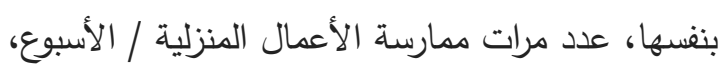

وقت النوم في اليوم روقت الوجبة الأخيرة قبل النوم كدلالئة

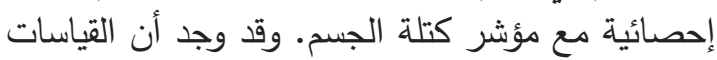

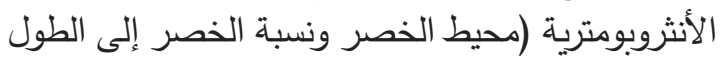

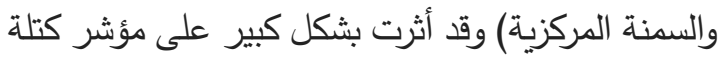

الجسم مما يعني أن هناك فرق كبير بين فئات السمنة

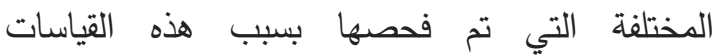

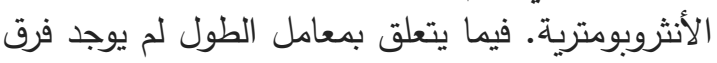

معنوي بين الوزن الزائد. وكذلك فئة السئة ابعضئهما

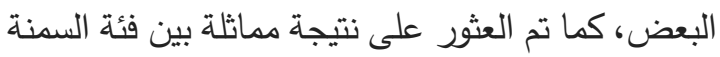

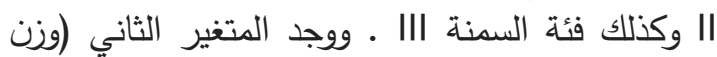

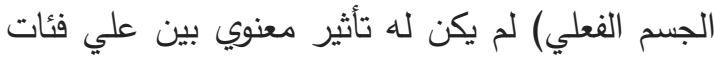

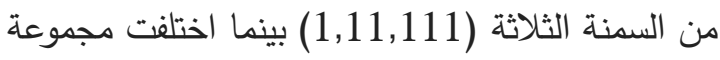

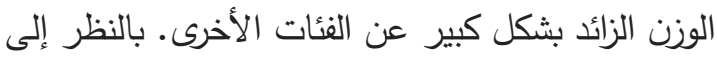

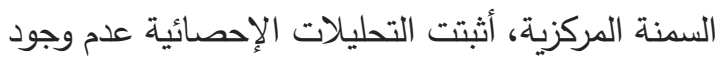

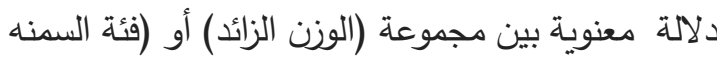

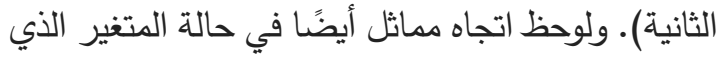
يسمى (محيط الورك) ولكن هذا غير معنوي كان بين فئة فئة

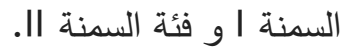

أجريت هذة الدراسة لتقييم الحالة التغذوية والسمنة

لربات البيوت اللائي (تتراوح أعمارهن بين 20 و 60

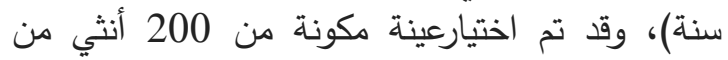

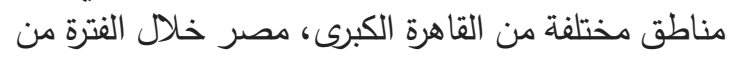

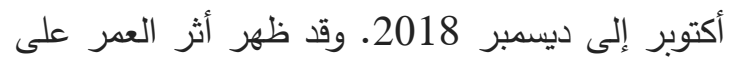
مؤشر كتلة الجسم بشكل كبير لمختلف فئات السمنة التي تم فحصها كما تم الكشف عن نتائج مماثلة في عامل

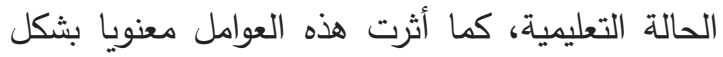

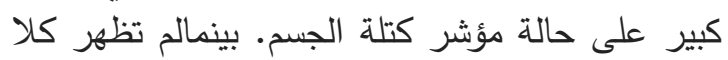

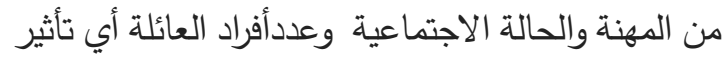

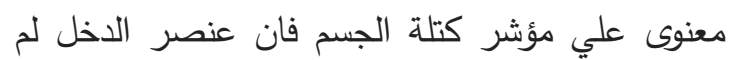

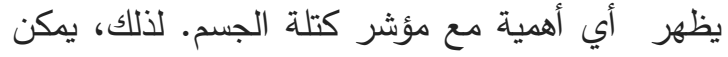

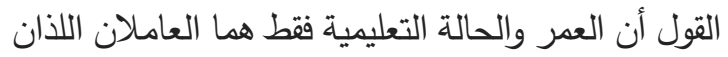

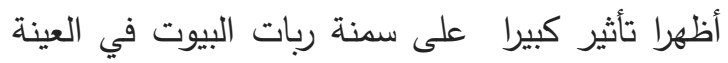

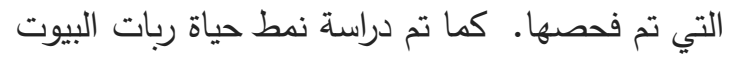

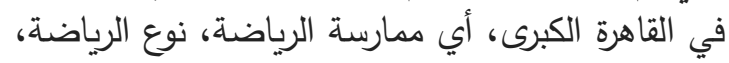

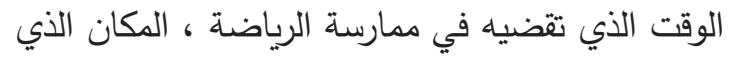

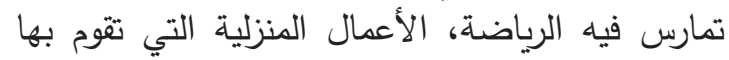

